\title{
Polarization coupling and pattern selection in a type-II optical parametric oscillator
}

\author{
Gonzalo Izús, ${ }^{*}$ Maxi San Miguel, and Daniel Walgraef ${ }^{\dagger}$ \\ Instituto Mediterráneo de Estudios Avanzados, IMEDEA (CSIC-UIB), ${ }^{*}$ Universitat de les Illes Balears, \\ E-07071 Palma de Mallorca, Spain
}

(Received 1 December 2001; published 30 September 2002)

\begin{abstract}
We study the role of a direct intracavity polarization coupling in the dynamics of transverse pattern formation in type-II optical parametric oscillators. Transverse intensity patterns are predicted from a stability analysis, numerically observed, and described in terms of amplitude equations. Standing wave intensity patterns for the two polarization components of the field arise from the nonlinear competition between two concentric rings of unstable modes in the far field. Close to threshold a wavelength is selected leading to standing waves with the same wavelength for the two polarization components. Far from threshold the competition stabilizes patterns in which two different wavelengths coexist.
\end{abstract}

DOI: 10.1103/PhysRevE.66.036228

PACS number(s): 42.65.Sf, 42.65.Yj, 42.50.-p

\section{INTRODUCTION}

Pattern formation is a ubiquitous manifestation of nonlinearity $[1,2]$, which presents specially interesting features in nonlinear optical systems [3-5]. The search for transverse structures in nonlinear optical systems is actively pursued for several reasons that include their possible application in alloptical signal processing and the investigation of macroscopic manifestations of quantum phenomena. These structures are the result of the interaction of nonlinearity and diffraction in transverse spatially extended devices such as nonlinear optical cavities of a large Fresnel number. Among the nonlinear systems analyzed, optical parametric oscillators (OPOs) have received a lot of attention from the theoretical viewpoint. Available results include the analysis of pattern formation [6-10], noise sustained structures [11], domain walls [12-14], and localized structures [15-18]. A growing interest in these transverse structures in OPOs arise also from the study of quantum spatial correlations present in these patterns [5,19-21]. Transverse patterns in OPOs have been recently observed in nonplane resonators [22].

In an OPO two first harmonic (FH) fields (signal and idler) are generated inside the crystal by parametric downconversion of the external pump field. In a type-I OPO, signal and idler fields have the same state of linear polarization. In a type-II OPO, they are orthogonally polarized. This polarization degree of freedom can be used for a nonlinear construction of new states of the emitted light. For example, by means of a direct polarization coupling produced by an intracavity quarter wave plate $(\lambda / 4$ plate), it is possible to produce $[23,24]$ states in which the signal and idler are degenerate in frequency, and phase locked. A general question

\footnotetext{
*Permanent address: Departamento de Física, Facultad de Ciencias Exactas y Naturales, Universidad Nacional de Mar del Plata y CONICET, Funes 3350 (7600) Mar del Plata, Argentina. Electronic address: izus@mdp.edu.ar

†Permanent address: Center for Nonlinear Phenomena and Complex Systems, Université Libre de Bruxelles, Campus Plaine, Blvd. du Triomphe B.P 231, 1050 Bruxelles, Belgium.

\#URL: http://www.imedea.uib.es/PhysDept
}

that we address in this paper is the effect of this type of direct polarization coupling in the problem of transverse pattern formation in a type-II OPO.

When considering transverse spatial degrees of freedom in a type-II OPO without a direct polarization coupling, there are two different regimes. In one of them, characterized by a positive effective detuning, homogeneous solutions are selected at threshold with an arbitrary relative phase between the signal and idler fields. For effective negative detuning a finite wave number is selected at threshold and a phase pattern-traveling wave (TW) - is asymptotically selected for each FH field, while the intensity remains homogeneous $[25,26]$. The effects of direct polarization coupling between the signal and idler in a type-II OPO for a positive effective detuning was discussed in Refs. [27,28]: Spatial domains of equivalent, but different, self-phase-locked homogeneous solutions appear. They are separated by phase polarization domain walls. These walls are generally Bloch walls whose motions lead to complex spatiotemporal states. In this paper we consider the situation of negative effective detuning in order to characterize the way in which polarization coupling modifies the process of pattern formation. In particular, one expects that the coupling between the signal and idler can generate standing waves (SWs), i.e., stripe intensity patterns, from the TWs that exist for the signal and idler when there is no direct polarization coupling.

For a type-II OPO with an intracavity $\lambda / 4$ plate and for FH's negative detunings, we predict, and numerically confirm, that there is a threshold of pattern formation above which the FH's far fields exhibit, during the transient dynamics, two concentric rings of growing unstable modes. Therefore, the coupling does not only lead from TWs to SWs, but it introduces two different wavelengths, giving rise to an interesting problem of wavelength competition and pattern selection. The values of the different wavelengths are controlled by the $\lambda / 4$ plate polarization coupling. In a "symmetric case" (i.e., all the dynamical parameters for the signal and idler are equal) we show that the two wavelengths coexist for long times. Real and imaginary parts of each FH field present in this case SW patterns with different wavelengths which consist of domains of stripe patterns. For asymmetric FH's coefficients and near threshold, we show that one of the 


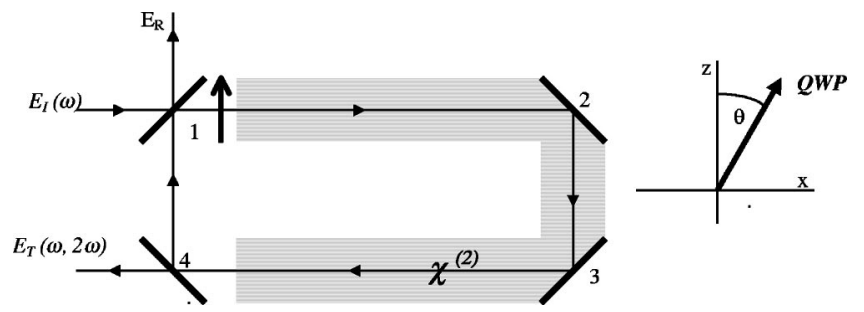

FIG. 1. (Left) Ring cavity filled with a passive quadratic medium. Mirrors 1 and 4 have transmission coefficient $T \ll 1$, mirrors 2 and 3 have $100 \%$ reflectivity. $E_{I}, E_{R}$, and $E_{T}$ are the incident, reflected, and transmitted fields, respectively. Patterns are predicted in the transverse plane to propagation. The arrow indicates the intracavity quarter wave plate. (Right) The angle $\theta$ between the quarter wave plate's fast axis and the crystal's principal axis is indicated.

wavelengths dominates and intensity stripe patterns (standing waves) with the same wavelength emerge for the signal and idler. Far from threshold the dynamics is similar to the symmetric case. This transition from wavelength coexistence to dominance of one of the two competing wavelengths is described by means of an amplitude equation analysis. The amplitude equations give a full description of the observed dynamics.

The paper is organized as follows. Section II reviews the mean-field equations for this system. In Sec. III, we analyze the threshold for pattern formation. The instability is characterized in terms of the eigenfunctions associated with the critical modes. In Sec. IV, we discuss numerically the dynamics of transverse pattern. In Sec. V, we derive amplitude equations for the critical modes, which give a clear interpretation of the observed dynamics. We summarize our main conclusions in Sec. VI.

\section{MEAN-FIELD EQUATIONS}

We consider an optical parametric oscillator that consists of a ring optical cavity filled with a birefringent, nonlinear quadratic medium, and is externally pumped by a uniform laser beam. A direct polarization coupling between the FH fields, which take into account the effect of an intracavity quarter wave plate, is also included in the model (see Fig. 1). This wave plate provides a polarization mixing between the signal $A_{x}$ and idler $A_{y}$ fields $[23,24]$. The signal and the idler can be either frequency degenerate or nondegenerate, depending on the frequency selection rules imposed by the combined effects of the parametric down-conversion, the cavity resonances, and the phase matching [29-31]; but they are always polarization non-degenerate (type-II interaction). In the mean-field approximation, and considering the paraxial and the single longitudinal mode approximation for all the fields, the equations describing the time evolution for the linear polarization components of the second harmonic (SH) $\left[B_{x, y}(x, y, t)\right]$ and the FH $\left[A_{x, y}(x, y, t)\right]$ slowly varying envelopes of the electric fields in a type-II OPO are [24,28]

$$
\begin{aligned}
\partial_{t} B_{x}= & \gamma_{x}^{\prime}\left[-\left(1+i \Delta_{x}^{\prime}\right) B_{x}+i \alpha_{x}^{\prime} \nabla^{2} B_{x}+2 i K_{0} A_{x} A_{y}+E_{0}\right. \\
& \left.+c^{\prime} B_{y}\right],
\end{aligned}
$$

$$
\begin{gathered}
\partial_{t} B_{y}=\gamma_{y}^{\prime}\left[-\left(1+i \Delta_{y}^{\prime}\right) B_{y}+i \alpha_{y}^{\prime} \nabla^{2} B_{y}-c^{\prime *} B_{x}\right], \\
\partial_{t} A_{x}=\gamma_{x}\left[-\left(1+i \Delta_{x}\right) A_{x}+i \alpha_{x} \nabla^{2} A_{x}+i K_{0} A_{y}^{*} B_{x}+c A_{y}\right], \\
\partial_{t} A_{y}=\gamma_{y}\left[-\left(1+i \Delta_{y}\right) A_{y}+i \alpha_{y} \nabla^{2} A_{y}+i K_{0} A_{x}^{*} B_{x}-c^{*} A_{x}\right],
\end{gathered}
$$

where with no loss of generality we take $A_{x}, B_{x}$ as ordinary polarized beams and $A_{y}, B_{y}$ as extraordinary polarized [26]. The coefficients $\gamma_{x, y}, \gamma_{x, y}^{\prime}$ (cavity decay rates); $\Delta_{x, y}, \Delta_{x, y}$, and $\Delta_{x, y}^{\prime}$ (cavity detunings); and $\alpha_{x, y}, \alpha_{x, y}^{\prime}$ (diffraction coefficients) are defined as in Refs. [6,27]. Due to the birefringence of the nonlinear crystal, all the diffraction coefficients can be slightly different, even when the signal and idler are frequency degenerate. Second harmonic cavity detunings can include corrections for a possible phase mismatch $\Delta K$ of the parametric interaction. In order to be consistent with the mean-field limit, we require that $\Delta K \ll 1 / L$, where $L$ is the cavity length $[11,31]$. Other parameters are the nonlinearity $K_{0}$ and the injected pump $E_{0}$ (bifurcation parameter) that, for the sake of simplicity, we take as real and polarized along the same direction than the phase-matched component of the second harmonic field $B_{x}$. Hence, $B_{y}$ neither is pumped nor is nonlinearly coupled with other components of the field. In Eqs. (1) the direct polarization coupling constants $\left(c, c^{\prime}\right)$ account for the effects produced by the $\lambda / 4$ plate and they are related to the phase mismatch and the axes of the $\lambda / 4$ plate by

$$
c \sim \sin (2 \phi) \exp (i \xi)
$$

where $\phi$ is the angle between the $\lambda / 4$ plate's fast axis and the principal axis of the crystal. The phase $\xi$ is the round-trip phase shift between the signal and idler at frequency degeneracy (or between $B_{x}$ and $B_{y}$ for $c^{\prime}$ ). The coupling strength $|c|$ depends on a number of factors, including mode matching and Poynting vector walkoff [23]. Here we assume propagation along the optical axis (i.e., we neglect spatial walk-off). We focus on the effects produced by the rotation angle $\phi$, which is an important experimental parameter to control the effects described below. We note that a similar coupling is associated with an intracavity $\lambda / 2$ plate [32]. Finally other forms of similar linear coupling terms between the signal and idler considered in Refs. [27,28] are associated with a birefringent and/or dichroic cavity mirror in a type-II OPO.

The linear coupling $c$ breaks the phase invariance that Eqs. (1) have for $c=0$ under changes of the relative phase of the FH fields: $\left[A_{x}, A_{y}\right] \rightarrow\left[\exp (i \varphi) A_{x}, \exp (-i \varphi) A_{y}\right]$. However, the phase of the signal and idler fields can be adjusted in order to include the phase of $c$ in the FH fields; i.e, given $c=|c| \exp (i \xi), \quad$ the transformation $\left[A_{x}, A_{y}\right] \rightarrow\left[\hat{A}_{x}, \hat{A}_{y}\right]$ $=\left[\exp (-i \xi / 2) A_{x}, \exp (i \xi / 2) A_{y}\right]$ leaves Eqs. (1) unchanged except for the replacement $c \rightarrow|c|$. For the sake of clarity, we will then present our main results for $c$ real. The generalization for $c$ complex is trivial using the phase transformation of the FH fields just described. 


\section{LINEAR STABILITY ANALYSIS}

\section{A. Threshold analysis}

In this section, we present the linear stability analysis of the steady-state solution of Eqs. (1) corresponding to the OPO operating below the threshold of signal generation. This trivial uniform steady state (off state) is

$$
\begin{gathered}
A_{x}=A_{y}=0 \\
B_{x}=\left(1+i \Delta_{y}^{\prime}\right) E_{0} /\left[1-\Delta_{x}^{\prime} \Delta_{y}^{\prime}-\left|c^{\prime}\right|^{2}+i\left(\Delta_{x}^{\prime}+\Delta_{y}^{\prime}\right)\right] \\
B_{y}=-c^{\prime} * B_{x} /\left(1+i \Delta_{y}^{\prime}\right) .
\end{gathered}
$$

The threshold for transverse pattern formation is determined by linearizing Eqs. (1) around this solution and looking for instabilities. The steady state becomes unstable only along the directions of the FH components $\left(A_{x}, A_{y}\right)$ of the eigenvectors, and thus the analysis reduces to the study of two linearly coupled complex equations. Because of the complex nature of the field variables, it is convenient to consider the real and the imaginary parts of these equations for each $\mathrm{FH}$ field. The most general solution of the perturbations is hence given by a linear superposition of terms of the form $\left[\operatorname{Re}\left(A_{x, y}\right), \operatorname{Im}\left(A_{x, y}\right)\right] \sim \exp [i \vec{q} \cdot \vec{r}+\lambda(\vec{q}) t]$, where $\lambda(\vec{q})$ is the growth rate of the perturbations and $\vec{q}$ is its transverse wave vector.

For $c=c^{\prime}=0$, the linear stability analysis shows that the trivial solution is stable for $|F|<\left|F_{c}\right|$, where $F$ is a normalized pump intensity

$$
F=K_{0} E_{0} /\left(1+i \Delta_{x}^{\prime}\right) .
$$

For $\widetilde{\Delta}=\gamma_{x} \Delta_{x}+\gamma_{y} \Delta_{y}>0$ the most unstable mode corresponds to an homogeneous solution $q_{0}=0$. In this paper, we focus on the case $\widetilde{\Delta}<0$ for which the unstable modes at threshold $\left(\left|F_{c}\right|=1\right)$ correspond to transverse traveling waves $A_{x}, A_{y}^{*} \simeq \exp [i \vec{q} \cdot \vec{r}+\lambda(\vec{q}) t]$, whose two-dimensional wave vector $\vec{q}$ lies on a circle centered at 0 with radius $q_{0}$ $=\sqrt{-\tilde{\Delta} / \tilde{\alpha}}$, where $\tilde{\alpha}=\gamma_{x} \alpha_{x}+\gamma_{y} \alpha_{y}$. For $|F|>\left|F_{c}\right|$ and $\tilde{\Delta}$ $<0$, any $\vec{q}$ mode on the circle, and the opposite mode for the orthogonal component of the field, can be selected at threshold by means of spontaneous symmetry breaking. Hence, a phase pattern appears above threshold for $A_{x}$ and $A_{y}$ (traveling waves) with opposite wave vectors, while the intensity remains homogeneous in both polarizations [25,26]. It should be noted that when idler and signal fields are degenerate both in frequency and polarization, i.e., degenerate type-I (OPO) (DOPO), Eqs. (1) must be solved with the further condition $A_{x}=A_{y}$. In this case, standing waves states are selected at threshold [6]. The linear coupling of $A_{x}$ with $A_{y}$ considered in this paper is expected to produce standing waves for each polarization component also in a type-II OPO.

For $c \neq 0$ the threshold for pattern formation remains at $\left|F_{c}\right|=1$, but a main difference is that now the wave vectors of the most unstable modes lie on two concentric circles of different radii. This gives rise to a wave number competition in the process of pattern formation, as shown below. Closed expressions are hard to obtain in the general case, but for $\gamma_{x}=\gamma_{y}=\gamma$ the two eigenvalues of the linearized equations that characterize the instability are

$$
\lambda_{1,2}=\gamma\left[-1+\frac{1}{2} \sqrt{4|F|^{2}-4|c|^{2}-2\left(\Theta_{x}^{2}+\Theta_{y}^{2}\right) \pm 2 \Upsilon}\right],
$$

where we have defined

$$
\begin{gathered}
\Theta_{j}=\Delta_{j}+\alpha_{j} q^{2}(j=x, y), \\
\Upsilon=\sqrt{-4|F|^{2}\left(\Theta_{x}-\Theta_{y}\right)^{2}+4|c|^{2}\left(\Theta_{x}+\Theta_{y}\right)^{2}+\left(\Theta_{x}^{2}-\Theta_{y}^{2}\right)^{2}}
\end{gathered}
$$

and we have introduced the normalized pump amplitude

$$
F=\left(1+i \Delta_{y}^{\prime}\right) K_{0} E_{0} /\left[1-\Delta_{x}^{\prime} \Delta_{y}^{\prime}-\left|c^{\prime}\right|^{2}+i\left(\Delta_{x}^{\prime}+\Delta_{y}^{\prime}\right)\right] \text {, }
$$

which coincides with $F$ given by Eq. (4) for $c^{\prime}=0$. Two other eigenvalues remain always negative. Note that the normalized form of $F$ [Eq. (7)] implies that the linear coupling between the two components of the second harmonic field modifies the instability threshold even when $B_{y}$ is resonant.

To avoid cumbersome expressions, analytical results are derived in this section for the particular case $\gamma_{x}=\gamma_{y}=\gamma$, $\alpha_{x}=\alpha_{y}=\alpha$, and $\Delta_{x}=\Delta_{y}=\Delta(<0)$. In this case, the eigenvalues $\lambda_{1,2}(\vec{q})$ become

$$
\lambda_{1,2}=\gamma\left[-1+\sqrt{|F|^{2}-\left(\Delta+\alpha|\vec{q}|^{2} \pm c\right)^{2}}\right]
$$

where the plus (minus) sign corresponds to $\lambda_{1}\left(\lambda_{2}\right)$. From Eq. (8) we get the threshold of instability for perturbations with an arbitrary wave vector $\vec{q}$ :

$$
\left|F_{1,2}(c)\right|^{2}=1+\left[\Delta+\alpha|\vec{q}|^{2} \pm c\right]^{2},
$$

where the plus (minus) sign corresponds to $F_{1}\left(F_{2}\right)$. Therefore, for $c<-\Delta$ the instability takes place at the critical threshold $\left|F_{c}\right|=1$ and the unstable modes at threshold correspond to transverse traveling waves whose two-dimensional real wave vector $\vec{q}$ lies on either of two concentric circles, centered at 0 with radius $q_{1,2}$ :

$$
q_{1,2}^{2}=\left|\vec{q}_{1,2}\right|^{2}=\frac{-\Delta \mp c}{\alpha} .
$$

In Fig. 2, we show the instability threshold for perturbations of different wave numbers. The threshold $F_{c}=1$ is the same for $c=0$ and $c \neq 0$, but for $c \neq 0$ the instability takes place at two different wavevectors of modulus $q_{1,2}$ indicated in the figure. Homogeneous perturbations $(\vec{q}=0)$ have a larger instability threshold. The homogeneous phase-locked solutions associated with this threshold are discussed in the Appendix. We will not consider here the case $c>-\Delta>0$ for which at $F_{c}=1$ only the mode $q_{2}$ becomes unstable. In this case the mode $q_{1}=0$ becomes unstable for larger values of the pump. 


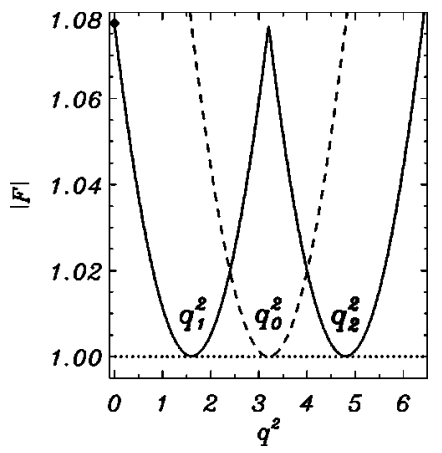

FIG. 2. Scaled threshold of instability $F$ for the trivial stationary solution [Eq. (3)] as a function of $q^{2}$ for the case of symmetric FH's coefficients. Solid (dashed) curve gives the threshold value of $F$ for $c \neq 0(c=0)$. The critical threshold $\left|F_{c}\right|=1$ is indicated as a dotted line. The instability takes place at two different wave vectors indicated by $q_{1}$ and $q_{2}\left(q_{0}\right.$ denotes the unstable wave vector for $c$ $=0)$. Parameters are $K_{0}=1, \Delta_{x, y}=-0.8, \Delta_{x, y}^{\prime}=0, \alpha_{x, y}=0.25$, $\alpha_{x, y}^{\prime}=0.125, c=0.4$, and $c^{\prime}=0.01$. The diamond indicates the threshold of instability for homogeneous perturbations $(\vec{q}=0)$.

The values of the most unstable wave numbers $q_{1}$ and $q_{2}$ depend on the absolute value of $c$ as follows from Eq. (10) (Fig. 3). In particular, $q_{1,2}^{2}$ coincide with $q_{0}^{2}$ for $c=0$ and vary linearly with $|c|$. The polarization coupling splits the circle of unstable modes for $c=0$ in two circles. The value of $c$ controls the magnitude of the split. We remark that for $c$ $\sim|\Delta|,\left|\vec{q}_{1}\right| \sim 0$ and patterns with very large wavelengths can be expected. For $|F|>\left|F_{c}\right|$ there is a band of unstable modes associated with each eigenvalue. In Fig. 4, we show the real part of the eigenvalues $\lambda_{1,2}$ as a function of $|\vec{q}|$ for the critical case $\left(|F|=\left|F_{c}\right|=1\right)$ and for one case above threshold. All the modes with $\operatorname{Re}\left(\lambda_{1,2}\right)>0$ are linearly unstable. The wave vectors with modulus $\left|\vec{q}_{1}\right|$ or $\left|\vec{q}_{2}\right|$ have the same maximum growth rate: $\lambda_{1,2}\left(\vec{q}_{1,2}\right)=\gamma(-1+|F|)$.

When the damping, detuning, or diffraction parameters for the signal and idler are different, the competing modes of wave numbers $q_{1}$ and $q_{2}$ have different thresholds. It follows from the numerical analysis of Eq. (5) that the smaller wave number $q_{1}$ becomes first unstable at $\left|F_{c}\right|=1$. In Fig. 5(a), we show the growth rate for perturbations of the trivial state as a

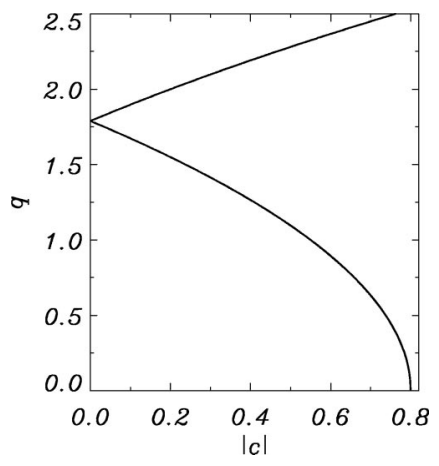

FIG. 3. Wave number of the critical unstable modes as a function of $|c|$. The lower branch corresponds to $q_{1}$ and the upper one to $q_{2}$. Parameter values as in Fig. 2.

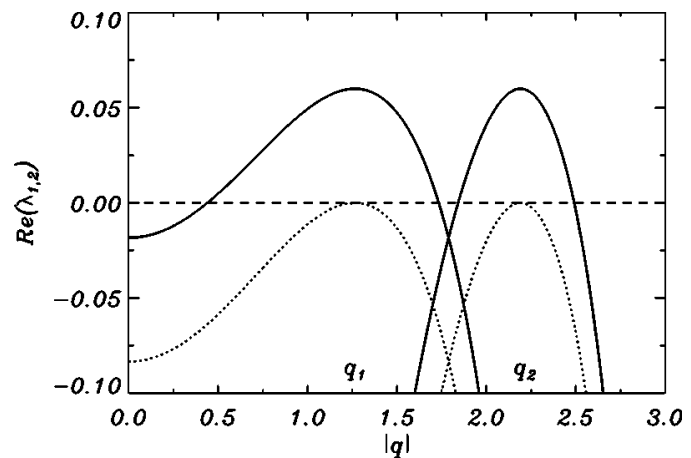

FIG. 4. Real part of the eigenvalues $\lambda_{1,2}$ as a function of $|\vec{q}|$ for the case of symmetric FH's coefficients. The solid lines correspond to $F=1.06$ and the dotted to criticality $F=F_{c}=1$. Left branches correspond to $\lambda_{1}$ and the right ones to $\lambda_{2}$. Here $\gamma_{x, y}=\gamma_{x, y}^{\prime}=1$ and the values of the other parameters are the same as in Fig. 2. The most unstable modes $q_{1}$ and $q_{2}$ correspond to the maximum of each line. The level $\operatorname{Re}\left(\lambda_{1,2}\right)=0$ is also indicated as a dashed horizontal line as a reference.

function of $|\vec{q}|$ for a case in which $|F|=1.0019$. The unstable mode of smaller wave number $q_{1}$ dominates in the linear regime. The difference in growth rates for $q_{1}$ and $q_{2}$ depends on the pumping level. For larger values of the pump, the growth rates of both unstable modes are of the same order, being the growth rate of $q_{1}$ larger than the one of $q_{2}$ [see Fig.
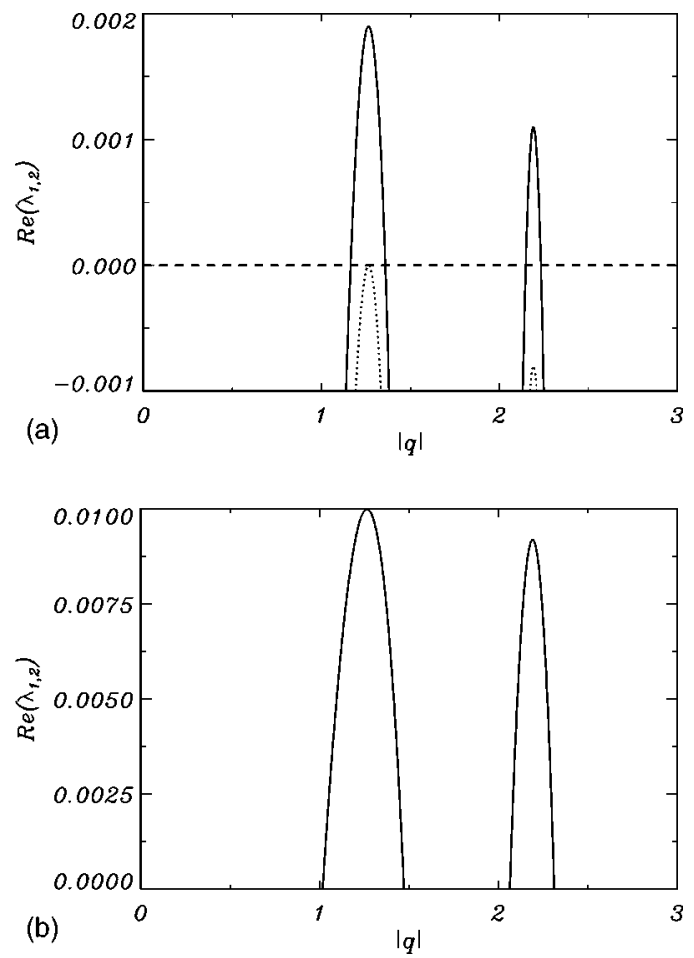

FIG. 5. Growth rate of the unstable modes as a function of $|\vec{q}|$ in a case in which the FH's coefficients are different. (a) Near threshold: $F=1.0019$ (solid line) and $F=1$ (dotted line). (b) Far from threshold: $F=1.02$. Coefficients values are $K_{0}=1, \Delta_{x, y}=-0.8$, $\Delta_{x, y}^{\prime}=0, \quad \gamma_{x}=\gamma_{x}^{\prime}=0.9901, \quad \gamma_{y}=\gamma_{y}^{\prime}=1.01, \quad \alpha_{x}=0.2475, \quad \alpha_{y}$ $=0.2525, \alpha_{x, y}^{\prime}=0.125, c=0.4$, and $c^{\prime}=0.01$. 
5(b)]. In the following section, we show that this fact deeply affects the nonlinear mode competition dynamics of the system.

We finally note that for asymmetric FH's coefficients, the critical modes have in general real eigenvalues [i.e., $\left.\operatorname{Im}\left(\lambda_{1,2}\left(\vec{q}_{1,2}\right)\right)=0\right]$. However, for very small values of $c$, i.e., when $\left|\vec{q}_{1}\right| \sim\left|\vec{q}_{2}\right|$, there is a small interval of values of the external pump $F$, which includes the critical value $F_{c}=1$, where $\operatorname{Im}\left(\lambda_{1,2}\left(\vec{q}_{1,2}\right)\right) \neq 0$. In this limit of $c \rightarrow 0$, the instability becomes convective, similarly to a situation considered in Ref. [33].

\section{B. Critical modes}

Next we consider some features of the early time dynamics of pattern formation that can be understood in terms of the eigenvectors corresponding to the eigenvalues of the linearized problem discussed above. First, we introduce the far field as the Fourier transform of the near field, where the near field is the transverse field configuration at the input/output cavity mirror. The far-field components $\widetilde{A}_{\vec{q}}(t)$ of $A_{x}$ (for example) are defined by

$$
A_{x}(\vec{r}, t)=\frac{1}{2 \pi} \int_{-\infty}^{\infty} \widetilde{A}_{\vec{q}}(t) \exp (i \vec{q} \cdot \vec{r}) d q_{x} d q_{y}
$$

In Fig. 6, we show numerical results [34] for a typical transverse profile of the $A_{x}$ and $A_{y}$ FH fields at an early time after the pump is increased beyond its threshold value. These results correspond to the case of symmetric coefficients in which two competing wave numbers have the same growth rate. In Fig. 6(a), we show the near field of the signal intensity pattern and its far field. The two concentric rings of the far field correspond to unstable wave vectors $\vec{q}$ with arbitrary orientation and a wave number around $q_{1}$ (inner ring) and $q_{2}$ (outer ring). The near field is the result of the interference among all the unstable modes of both rings in the far field. However, the interference takes place in such a way that the real part of $A_{x}$ is associated with the unstable modes of the outer ring, while its imaginary part is associated with the unstable modes of the inner ring. In addition, there is a high correlation between the transverse structures observed in the signal $A_{x}$ and idler $A_{y}$ fields. This is illustrated in Figs. 6(b)6(d). In Figs. 6(b) and 6(c), we show the near field of the real and imaginary parts of $A_{x}$ and $A_{y}$. It is observed that the real and imaginary parts of the field $A_{x}$ support transverse patterns with different wavelengths. The same fact is observed

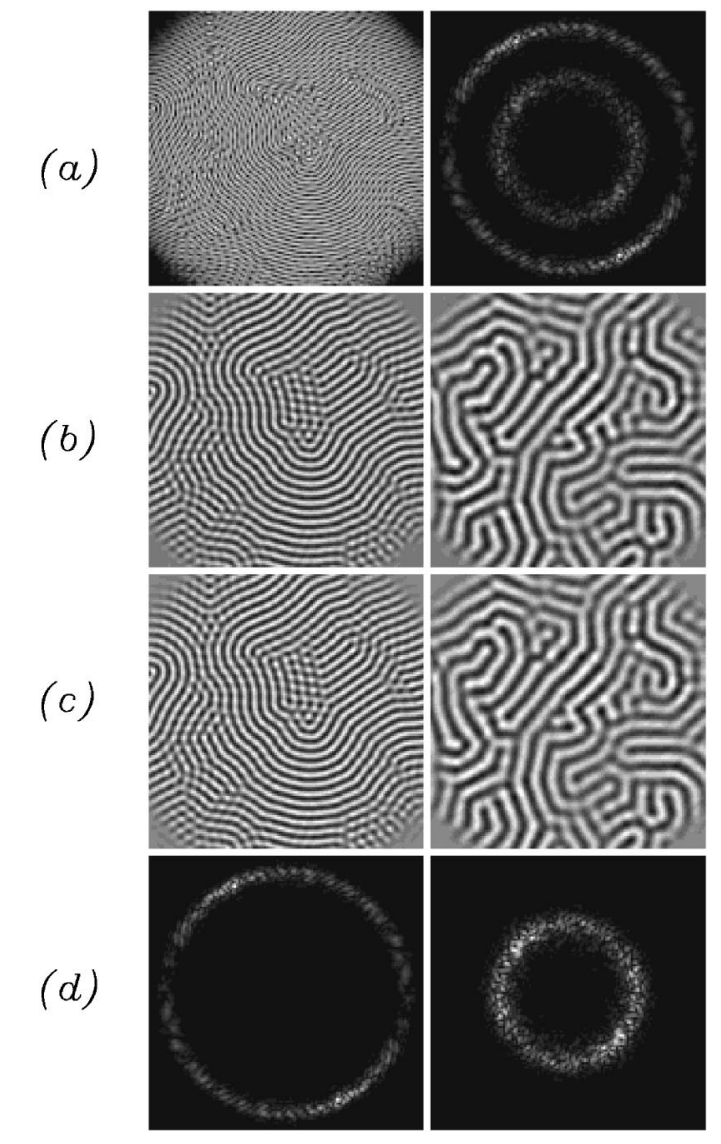

FIG. 6. A snapshot at $t=900$ of the FH's fields spontaneously generated from random initial conditions close to the trivial steady state given by Eq. (3). (a) Left: near field $\left|A_{x}\right|$; right: far field $\left|\widetilde{A}_{q}\right|$. (b) $A_{x}$ field. Left: $\operatorname{Re}\left(A_{x}\right)$; right: $\operatorname{Im}\left(A_{x}\right)$. (c) $A_{y}$ field. Left: $\operatorname{Im}\left(A_{y}\right)$; right: $\operatorname{Re}\left(A_{y}\right)$. (d) Absolute value of the Fourier transform. Left: $\operatorname{Re}\left(A_{x}\right)$. Right: $\operatorname{Im}\left(A_{x}\right)$. Parameters are $F=1.16, K_{0}=1, \Delta_{x, y}=$ $-0.8, \Delta_{x, y}^{\prime}=0, \gamma_{x, y}=\gamma_{x, y}^{\prime}=1, \alpha_{x, y}=0.25, \alpha_{x, y}^{\prime}=0.125, c=0.4$, and $c^{\prime}=0.01$.

in the idler field $A_{y}$, but real and imaginary parts have a different wavelength than for $A_{x}$. In fact we observe that $\operatorname{Re}\left(A_{x}\right) \simeq \operatorname{Im}\left(A_{y}\right)$, while $\operatorname{Im}\left(A_{x}\right) \simeq \operatorname{Re}\left(A_{y}\right)$. In Fig. 6(d), we show the far fields of $\operatorname{Re}\left(A_{x}\right)$ and $\operatorname{Im}\left(A_{x}\right)$. This gives evidence of the different wave number associated with $\operatorname{Re}\left(A_{x}\right)$ and $\operatorname{Im}\left(A_{x}\right)$. The two rings in the far fields of $\operatorname{Re}\left(A_{x}\right)$ and $\operatorname{Im}\left(A_{x}\right)$ correspond, respectively, to the outer and inner rings of Fig. 6(a).

These numerical facts can be explained in terms of the eigenvectors $\Lambda_{1,2}(\vec{q})$ associated with the eigenvalues $\lambda_{1,2}$ introduced in Eq. (8). They can be written as

$$
\begin{gathered}
{\left[\operatorname{Re}\left(A_{x}\right), \operatorname{Im}\left(A_{x}\right), \operatorname{Re}\left(A_{y}\right), \operatorname{Im}\left(A_{y}\right)\right]^{T}=\Lambda_{1}(\vec{q})=C_{1}\left[\kappa_{1}, 1,1,-\kappa_{1}\right]^{T} \exp (i \vec{q} \cdot \vec{r})} \\
\Lambda_{2}(\vec{q})=C_{2}\left[1,-\kappa_{2}, \kappa_{2}, 1\right]^{T} \exp (i \vec{q} \cdot \vec{r}),
\end{gathered}
$$




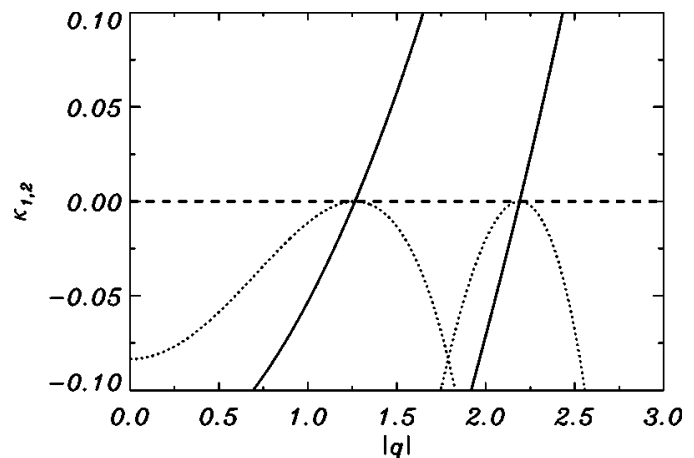

FIG. 7. Amplitudes $\kappa_{1,2}$ (solid line) as a function of $|\vec{q}|$. The real part of the critical eigenvalues $\lambda_{1,2}$ (dotted lines) and the zero level (dashed line) are also plotted as reference. Here $F=1$ and the values of the other parameters are the same as Fig. 4.

where we have introduced

$$
\begin{gathered}
\kappa_{1}=\frac{-\lambda_{1}+F-1}{\Delta+\alpha|\vec{q}|^{2}+c}, \\
\kappa_{2}=\frac{-\lambda_{2}+F-1}{\Delta+\alpha|\vec{q}|^{2}-c},
\end{gathered}
$$

and the normalization constants $C_{j}^{-1}=\sqrt{2\left(1+\left|\kappa_{j}\right|^{2}\right)}, j$ $=1,2$. For simplicity here we take $F$ to be real (i.e., resonant pump field). Exactly at threshold, $\kappa_{1,2}$ vanishes for the corresponding critical wave vector $\kappa_{1,2}\left(\vec{q}_{1,2}\right)=0$. The general dependence of $\kappa_{1,2}$ on the wave number at threshold is shown in Fig. 7. At threshold, $F=F_{c}=1$ and the eigenvectors $\Lambda_{1,2}(\vec{q})$ are damped for any $\vec{q}$ except $\Lambda_{1}\left(\vec{q}=\vec{q}_{1}\right)$ and $\Lambda_{2}\left(\vec{q}=\vec{q}_{2}\right)$ :

$$
\begin{aligned}
& \Lambda_{1}\left(\vec{q}_{1}\right)=\frac{1}{\sqrt{2}}[0,1,1,0]^{T} \exp \left(i \vec{q}_{1} \cdot \vec{r}\right), \\
& \Lambda_{2}\left(\vec{q}_{2}\right)=\frac{1}{\sqrt{2}}[1,0,0,1]^{T} \exp \left(i \vec{q}_{2} \cdot \vec{r}\right),
\end{aligned}
$$

which are marginal (zero growth rate) and define the direction in the functional space along which the instability takes place. The form of the eigenvectors (14) explains our numerical finding (Fig. 6) that at short times, when the linear approximation to the dynamics remains valid, the components of the FH's fields $\left[\operatorname{Re}\left(A_{x}\right), \operatorname{Im}\left(A_{y}\right)\right]$ and $\left[\operatorname{Im}\left(A_{x}\right), \operatorname{Re}\left(A_{y}\right)\right]$ only sustain patterns with a wave vector of modulus $\left|\vec{q}_{2}\right|$ or $\left|\vec{q}_{1}\right|$, respectively. As we discuss in the following section, this gives rise to pattern formation with competing wavelengths that can be rather different.

There is an interesting symmetry in the far-field components of the first harmonics fields. We focus in the $A_{x}$ field, but the discussion is also valid for $A_{y}$. Our numerical results indicate, as shown, for example, in Fig. 8, that the real (imaginary) part of the far-field component $\widetilde{A}_{q}(t)$ is an odd
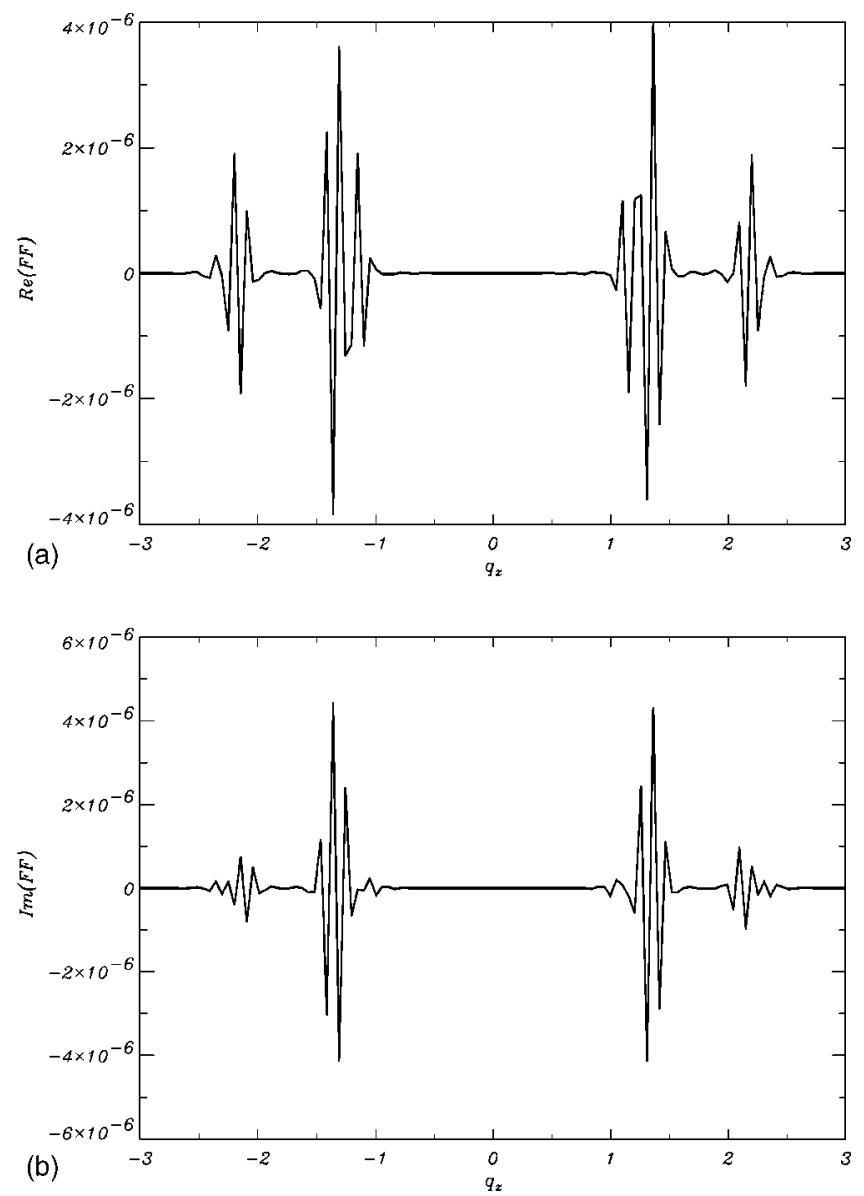

FIG. 8. A typical result for a cut of the far-field (FF) amplitude $\widetilde{A}_{q}(t)$ along the line $q_{y}=0$ for the $A_{x}$ field during the transient state. (a) Real part; (b) imaginary part. Parameters are $F=1.06, K_{0}=1$, $\Delta_{x, y}=-0.8, \quad \Delta_{x, y}^{\prime}=0, \quad \gamma_{x}=\gamma_{x}^{\prime}=0.9901, \quad \gamma_{y}=\gamma_{y}^{\prime}=1.01, \quad \alpha_{x}$ $=0.2475, \alpha_{x}=0.2525, \alpha_{x, y}^{\prime}=0.125, c=0.4$, and $c^{\prime}=0.01$.

function for $\vec{q}=\vec{q}_{1}$ and an even function for $\vec{q}=\vec{q}_{2}$ (even function for $\vec{q}=\vec{q}_{1}$ and an odd function for $\vec{q}=\vec{q}_{2}$ ). This property implies that

$$
\begin{gathered}
\widetilde{A}_{-\vec{q}_{1}}=-\widetilde{A}_{\underset{q_{1}}{*}}^{*}, \\
\widetilde{A}_{-\vec{q}_{2}}=\widetilde{A}_{\overrightarrow{q_{2}}}^{*} .
\end{gathered}
$$

Given that in the linear regime at threshold only excitations with wave vectors $\vec{q}_{2}$ or $\vec{q}_{1}$ contribute to the resulting structures in the real-valued fields $\operatorname{Re}\left(A_{x}\right)$ or $\operatorname{Im}\left(A_{x}\right)$, respectively, we find that the basic excitations for $\operatorname{Re}\left(A_{x}\right)$ and $\operatorname{Im}\left(A_{x}\right)$ are standing waves of the form

$$
\begin{aligned}
& \operatorname{Re}\left(A_{x}\right) \sim \widetilde{A}_{\vec{q}_{2}} \exp \left(i \vec{q}_{2} \cdot \vec{r}\right)+\widetilde{A}_{-\vec{q}_{2}} \exp \left(-i \vec{q}_{2} \cdot \vec{r}\right), \\
& i \operatorname{Im}\left(A_{x}\right) \sim \widetilde{A}_{\vec{q}_{1}} \exp \left(i \vec{q}_{1} \cdot \vec{r}\right)+\widetilde{A}_{-\vec{q}_{1}} \exp \left(-i \vec{q}_{1} \cdot \vec{r}\right)
\end{aligned}
$$

These standing waves are the interference between two opposite modes of the same ring of the far field that satisfy Eq. 
(a)
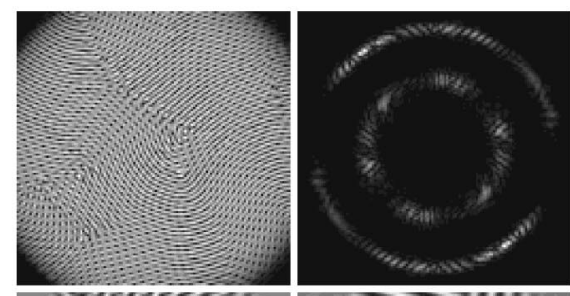

(b)
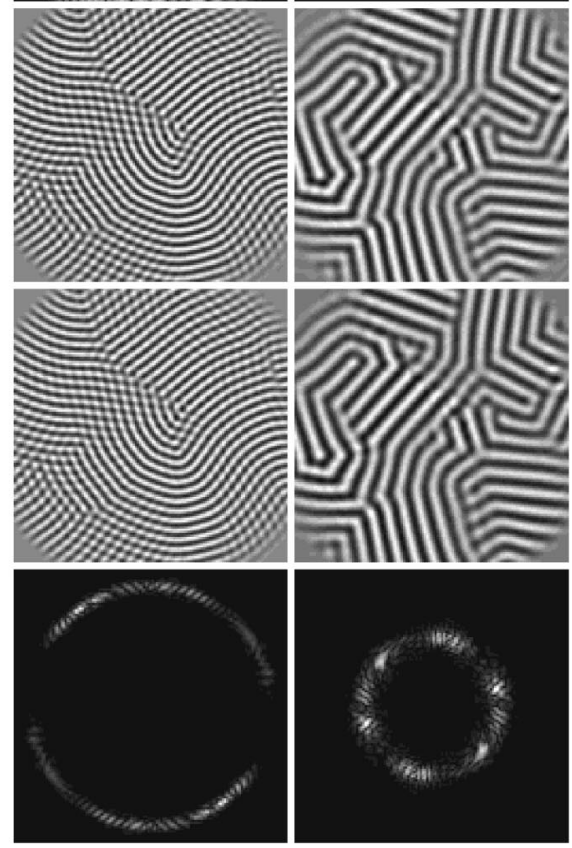

FIG. 9. Snapshots as in Fig. 6 at time $t=7800$. Parameters are the same as Fig. 6.

(15). Both modes have the same amplitude, but the global phase of the superposition of the two modes is different in each ring.

It is interesting to note that the circularly polarized components $A_{ \pm}$of the vectorial FH field give a natural description of the instability. We have already discussed that, at threshold, $\operatorname{Re}\left(A_{x}\right) \simeq \operatorname{Im}\left(A_{y}\right)$ and $\operatorname{Im}\left(A_{x}\right) \simeq \operatorname{Re}\left(A_{y}\right)$, so that $A_{+}=\left(A_{x}+i A_{y}\right) / \sqrt{2}=i \sqrt{2} \operatorname{Im}\left(A_{x}\right)$ and $A_{-}=\left(A_{x}-i A_{y}\right) / \sqrt{2}$ $=\sqrt{2} \operatorname{Re}\left(A_{x}\right)$. Therefore, it follows from Eq. (16) that the instability for $A_{+}\left(A_{-}\right)$takes place at $q_{1}\left(q_{2}\right)$. The two circularly polarized components will emerge at the instability as standing waves intensity patterns of different wave numbers. In fact, for symmetric coefficients and in the linearized version of Eqs. (1) around the trivial solution (3), $A_{+}$and $A_{-}$ are decoupled. They are nonlinearly coupled through the pump field $B_{x}$.

Our above discussion is for real values of the parameter $c$. When $c$ is complex $(\xi \neq 0)$, both the real and imaginary parts of $A_{x}$ and $A_{y}$ have contributions of unstable modes of wave numbers $q_{1}$ and $q_{2}$. Therefore, we observe transverse patterns with competing wavelengths in the real and the imaginary parts of $\hat{A}_{x, y}$. However, by changing the global phase of the FH fields, the problem can be considered in terms of $c$ real, as previously pointed out. (a)
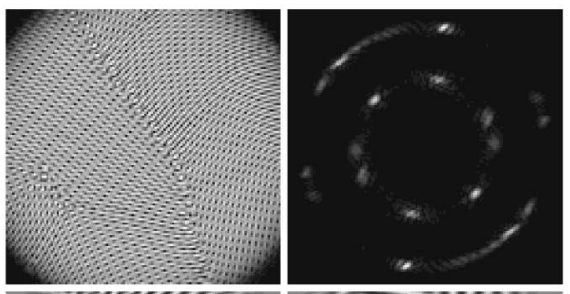

(b)
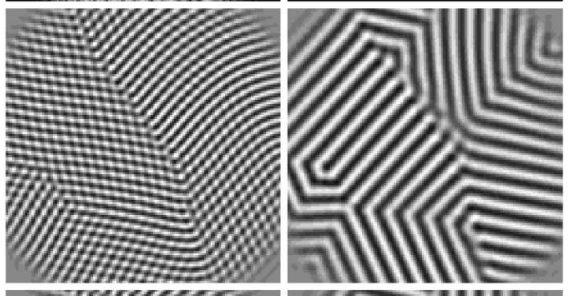

(c)
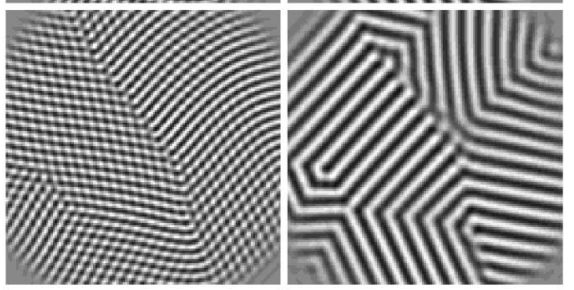

(d)
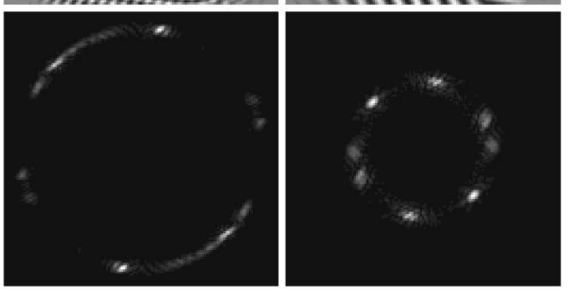

FIG. 10. Snapshots as in Fig. 6 at time $t=42800$. Parameters are the same as Fig. 6.

\section{TRANSVERSE INTENSITY PATTERNS}

In this section, we give a numerical description [34] of the patterns that are asymptotically selected after a regime of nonlinear competition among the unstable modes of wave numbers $q_{1}$ and $q_{2}$. A theoretical justification of these results is given in the following section in terms of an amplitude equation. We consider separately the cases of symmetric and nonsymmetric coefficients for the FH fields.

\section{A. Symmetric coefficients}

When $\gamma_{x}=\gamma_{y}=\gamma, \alpha_{x}=\alpha_{y}=\alpha$, and $\Delta_{x}=\Delta_{y}=\Delta$, the growth rates of the most unstable modes in the two circles of radii $q_{1}$ and $q_{2}$ are equal, and the instability takes place at both circles simultaneously (see Fig. 4). In this case the nonlinear competition keeps wave vectors in both circles in the far field excited for long times. The real and imaginary parts of the FH fields show patterns with different wavelengths, as discussed in Sec. III. To illustrate the dynamical evolution, we show in Figs. 9 and 10 snapshots of the pattern configuration at two different times. The two rings in the far field persist at long times. The pattern that appears in the longtime dynamics presents domains in which real and imaginary parts of each of the FH near fields show standing waves of arbitrary orientation and of different wavelengths for the real 
(a)

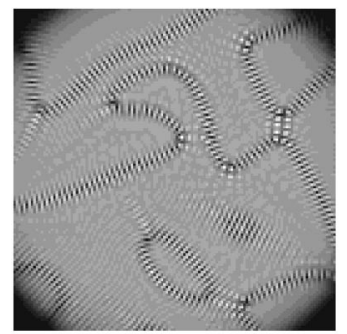

(c)

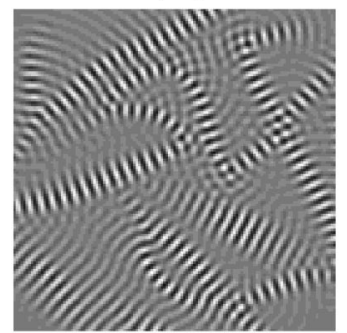

(b)

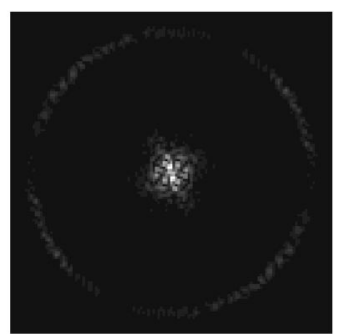

(d)

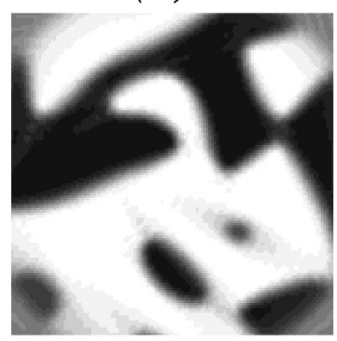

(a)
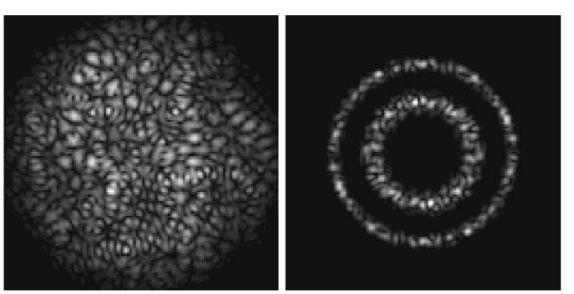

(b)
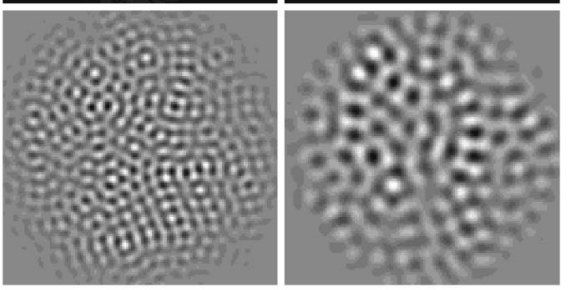

(c)
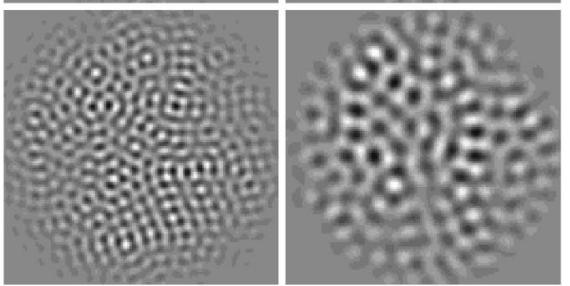

FIG. 11. A snapshot at time $t=3000$ of the field $A_{x}$ spontaneously generated from random initial conditions for a case in which the mode $\vec{q}=0$ is one of the most unstable modes. (a), (b), (c) and (d) show, respectively, the intensity, far field, real part and imaginary part of the signal field. In (c) and (d) patterns with very different wavelength can be appreciated. Parameter values are $F$ $=1.08, K_{0}=1, \Delta_{x, y}=-0.6, \quad \Delta_{x, y}^{\prime}=0, \quad \gamma_{x}=\gamma_{x}^{\prime}=\gamma_{y}=\gamma_{y}^{\prime}=1, \quad \alpha_{x}$ $=\alpha_{y}=0.25, c=0.6$ and $c^{\prime}=0.01$.

and imaginary parts. The arbitrary orientation of these standing waves comes from the spontaneous choice of two opposite wave vectors in the corresponding ring of the far field. Thus, the general picture is that there is long time competition among standing waves of different orientations and two different wave numbers.

A limiting situation is the case in which the inner ring collapses to the point $\vec{q}=0$. This situation takes place when $c=\left|\Delta_{x}\right|$. In this case, the growth of uniform domains occurs in one of the components of the FH vector field while the other component sustains standing waves of local arbitrary orientation, as shown in Fig. 11. This structure represents a case in which patterns and uniform domains coexist in the same complex field as an effect induced by the direct polarization coupling. This situation takes place near signal resonance for very small values of $c$.

\section{B. Nonsymmetric coefficients}

When the damping, diffraction, or detuning coefficients of the signal and idler are different, the nonlinear mode competition depends very much on how far above threshold the OPO is pumped. Near threshold, the relative difference in the magnitude of the growth rate of the unstable modes on the circles of wave numbers $q_{1}$ and $q_{2}$ is important, as follows from Fig. 5(a). This fact produces a strong change in the dynamics of the system. In Figs. 12, 13, and 14, we show snapshots of configurations at different times of the dynamical evolution. After a transient linear regime, discussed in
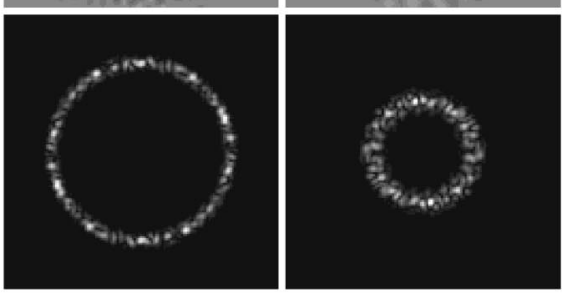

FIG. 12. Snapshots as in Fig. 6 at time $t=100$. Parameter values are $E_{0}=1.002, K_{0}=1, \Delta_{x, y}=-0.8, \Delta_{x, y}^{\prime}=0, \gamma_{x}=\gamma_{x}^{\prime}=0.9901$, $\gamma_{y}=\gamma_{y}^{\prime}=1.01, \alpha_{x}=0.2475, \alpha_{y}=0.2525, \alpha_{x, y}^{\prime}=0.125, c=0.4$, and $c^{\prime}=0.01$.

Sec. III and which is represented here by Fig. 12, there is a nonlinear competition between the two circles of unstable modes at intermediate times. This is shown in Fig. 13 where the far fields of $\operatorname{Re}\left(A_{x}\right)$ and $\operatorname{Im}\left(A_{y}\right)$ are seen to have competing contributions from the two circles. At late times the inner circle wins the competition and the final pattern is a standing wave of wave number $q_{1}$ both for $A_{x}$ and $A_{y}$. This nonlinear wave number selection can be traced back to the behavior of the growth rate, as shown in the following section. For long times there is also a spontaneous breaking of the rotational symmetry and a standing wave in an arbitrary direction is selected, as shown in Fig. 14. The real and imaginary parts of the FH fields show, in this case, patterns with the same wavelengths. The resulting structures originate in the interference between two arbitrary, but opposite, wave vectors of the inner circle. The resulting stripe intensity pattern is similar to that predicted for type-I DOPO in the sense that it is the interference between two opposite traveling waves [6]. However, physically, the energy and momentum conservation in the parametric down-conversion of pump photons only implies off-axis emission of idler and signal photons along two opposite directions without interference between them because they have orthogonal polarization. Due to polarization coupling, pure traveling waves are not 
(a)
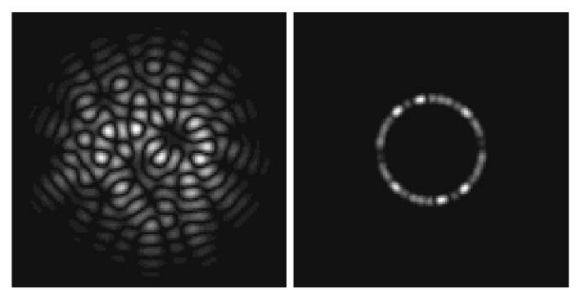

(b)
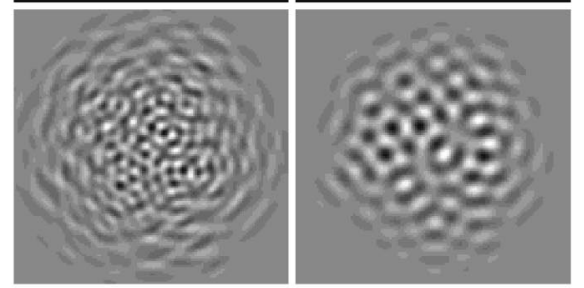

(c)
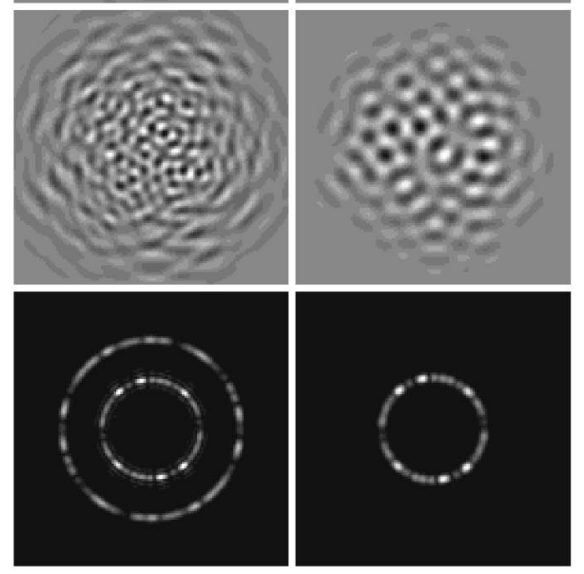

FIG. 13. Snapshots as in Fig. 12 at time $t=3200$.

solutions of Eqs. (1). The $\lambda / 4$ plate provides a mixing of polarization that allows the photons of the same $\mathrm{FH}$ field to interfere, producing a standing wave. This phenomenon is the same one that occurs in resonantly coupled complex Ginzburg-Landau equations [35,36]: the linear (polarization) coupling allows the formation of standing waves as the result of the interaction between opposite modes in the far field, in this case, the inner ring.

Far from threshold, the growth rates of the unstable modes $q_{1}$ and $q_{2}$ are of the same order [see Fig. 5(b)] and the dynamics of the system in this regime is equivalent to the symmetric case, as we prove in the following section. Therefore, in the asymmetric case the intensity of the external pump can be used to stabilize both rings of the far field which have a competing coexistence for long times and far from threshold. In Fig. 15, we show a typical long-time state for this regime.

\section{AMPLITUDE EQUATIONS}

Close to the instability threshold and using the general methods of nonlinear dynamics and pattern formation theory, one may derive amplitude equations for the patterns described numerically in the preceding section. It is now well known that equations are able to describe pattern evolution, selection, and stability. In particular, if, for simplicity, one (a)
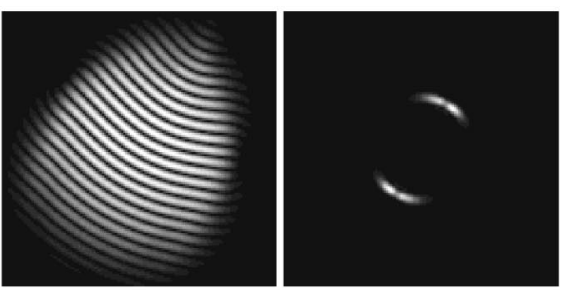

(b)
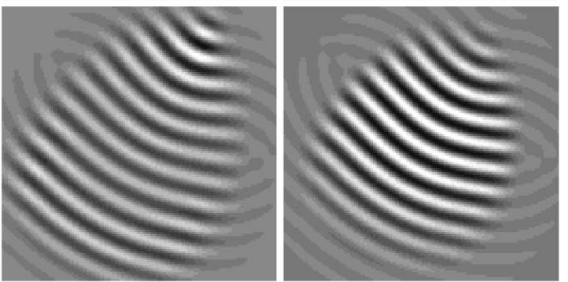

(c)
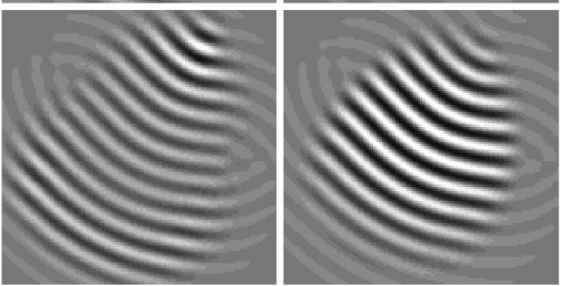

(d)
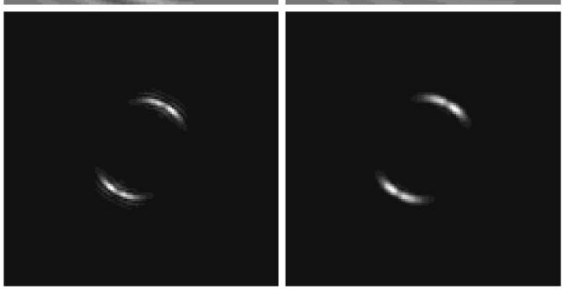

FIG. 14. Snapshots as in Fig. 12 at time $t=17600$. (a)

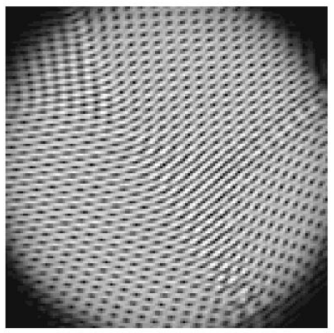

(c)

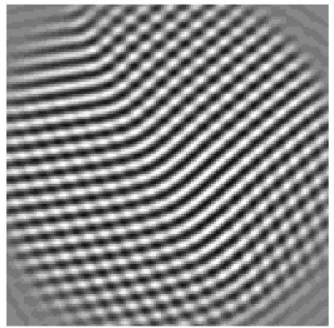

(b)

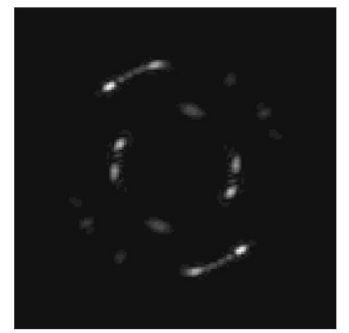

(d)

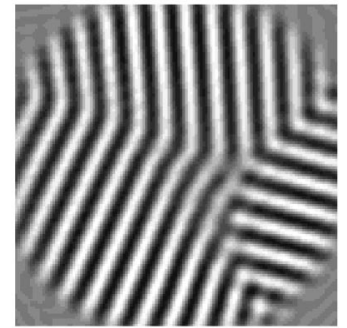

FIG. 15. A snapshot at time $t=50500$ of the field $A_{x}$ spontaneously generated from random initial conditions close to the trivial steady state in the asymmetric case. (a) (b), (c), and (d) show the intensity, far field, real part and imaginary part of $A_{x}$, respectively. Parameters are as in Fig. 12 except $F=1.06$. 
considers only the critical modes identified in Sec. III, field variables may by expressed as

$$
\left[\operatorname{Re}\left(A_{x}\right), \operatorname{Im}\left(A_{x}\right), \operatorname{Re}\left(A_{y}\right), \operatorname{Im}\left(A_{y}\right)\right]^{T}=A_{1}[0,1,1,0]^{T} \exp \left(i \vec{q}_{1} \cdot \vec{r}\right)+A_{-1}[0,1,1,0]^{T} \exp \left(-i \overrightarrow{q_{1}} \cdot \vec{r}\right)
$$

where $A_{i}(t)$ are slowly evolving amplitudes associated with the modes with wave vector $\vec{q}_{i}$. Given the relations (15), we have chosen here to define the amplitudes $A_{ \pm 1}$ and $A_{ \pm 2}$ as $\widetilde{A}_{ \pm q_{1}}=i A_{ \pm 1}$ and $\widetilde{A}_{ \pm \vec{q}_{2}}=A_{ \pm 2}$.

Standard analysis [2] leads to the following evolution equations for the symmetric case $\left(\Theta_{x}=\Theta_{y}\right.$ and $c^{\prime}=\Delta_{x}^{\prime}=0$ for simplicity):

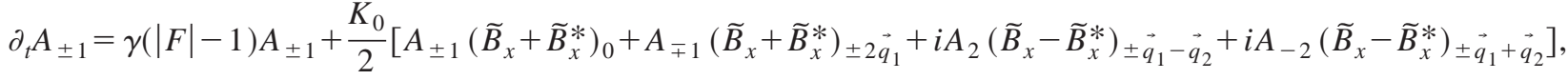

$$
\begin{aligned}
& \partial_{t} A_{ \pm 2}=\gamma(|F|-1) A_{ \pm 2}+\frac{K_{0}}{2}\left[A_{ \pm 2}\left(\widetilde{B}_{x}+\widetilde{B}_{x}^{*}\right)_{0}+A_{\mp 2}\left(\widetilde{B}_{x}+\widetilde{B}_{x}^{*}\right)_{ \pm 2 \vec{q}_{2}}+i A_{1}\left(\widetilde{B}_{x}-\widetilde{B}_{x}^{*}\right)_{ \pm} \vec{q}_{2}-\vec{q}_{1}+i A_{-1}\left(\widetilde{B}_{x}-\widetilde{B}_{x}^{*}\right)_{ \pm} \vec{q}_{1}+\vec{q}_{2}\right]
\end{aligned}
$$

where

$$
\partial_{t}\left(\widetilde{B}_{x}\right)_{q}=-\left(1+i \alpha^{\prime} q^{2}\right)\left(\widetilde{B}_{x}\right)_{q}+2 i K_{0}\left(\widetilde{A_{x} A_{y}}\right) \vec{q},
$$

where $\alpha^{\prime}=\alpha_{x}^{\prime}$ and $(\widetilde{\cdots})_{q}$ indicates Fourier transform. Using Eq. (19) and time scales separation near threshold $(|F|-1$ $\ll 1)$ one may write $\left(\widetilde{B}_{x}\right)_{j}=B_{j}$ as an expansion in the amplitudes $A_{i}$. One has, at lowest order,

$$
\begin{gathered}
B_{0}=-4 K_{0}\left(A_{1} A_{-1}+A_{2} A_{-2}\right), \\
B_{ \pm 2 \vec{q}_{i}}=-\frac{2 K_{0} A_{ \pm i} A_{ \pm i}}{1+4 i \alpha^{\prime} q_{i}^{2}}, \\
B_{ \pm \vec{q}_{2} \pm \vec{q}_{1}} \simeq 0 .
\end{gathered}
$$

This leads to

$$
\begin{aligned}
\partial_{t} A_{ \pm 1}= & \gamma(|F|-1) A_{ \pm 1}-4 K_{0}^{2} A_{ \pm 1}^{2} A_{\mp 1}-4 K_{0}^{2} A_{ \pm 1} A_{2} A_{-2} \\
& -4 \eta_{1} K_{0}^{2} A_{\mp 1} A_{ \pm 1}^{2}, \\
\partial_{t} A_{ \pm 2}= & \gamma(|F|-1) A_{ \pm 2}-4 K_{0}^{2} A_{ \pm 2}^{2} A_{\mp 2}-4 K_{0}^{2} A_{ \pm 2} A_{1} A_{-1} \\
& -4 \eta_{2} K_{0}^{2} A_{\mp 2} A_{ \pm 2}^{2},
\end{aligned}
$$

where

$$
\eta_{1,2}^{-1}=2\left[1+\left(4 \alpha^{\prime} q_{1,2}^{2}\right)^{2}\right] .
$$

Since $A_{-1}=A_{1}^{*}$ and $A_{-2}=A_{2}^{*}$, as follows from Eq. (15) and the definition of the amplitudes $A_{ \pm 1}$ and $A_{ \pm 2}$ after Eq. (17), one may finally write

$$
\begin{aligned}
& \partial_{t} A_{1}=\gamma(|F|-1) A_{1}-4 K_{0}^{2} A_{1}\left[\left(1+\eta_{1}\right)\left|A_{1}\right|^{2}+\left|A_{2}\right|^{2}\right], \\
& \partial_{t} A_{2}=\gamma(|F|-1) A_{2}-4 K_{0}^{2} A_{2}\left[\left(1+\eta_{2}\right)\left|A_{2}\right|^{2}+\left|A_{1}\right|^{2}\right] .
\end{aligned}
$$

These equations describe a weak competition between the amplitudes $A_{1}$ and $A_{2}$, so that the stable steady states of the system are given by

$$
\begin{aligned}
& \left|A_{1}\right|^{2}=\frac{\eta_{2}}{\eta_{1}+\eta_{2}+\eta_{1} \eta_{2}} \frac{\gamma(|F|-1)}{4 K_{0}^{2}}, \\
& \left|A_{2}\right|^{2}=\frac{\eta_{1}}{\eta_{1}+\eta_{2}+\eta_{1} \eta_{2}} \frac{\gamma(|F|-1)}{4 K_{0}^{2}} .
\end{aligned}
$$

These expressions are still valid when $\Delta^{\prime}$ and $c^{\prime}$ are different from zero, with $\eta_{1,2}$ given by

$$
\eta_{1,2}=\frac{1}{2} \frac{1+c^{\prime 2}+\left(\Delta^{\prime}+4 \alpha^{\prime} q_{1,2}^{2}\right)^{2}}{\left[1+\left(c^{\prime}+\Delta^{\prime}+4 \alpha^{\prime} q_{1,2}^{2}\right)^{2}\right]\left[1+\left(c^{\prime}-\Delta^{\prime}-4 \alpha^{\prime} q_{1,2}^{2}\right)^{2}\right]} .
$$

The asymptotic states of the dynamics, Eqs. (23), correspond to patterns built on two wave vectors $\vec{q}_{1}$ and $\vec{q}_{2}$, as confirmed by the numerical results of the preceding section. However, when the kinetic coefficients of $x$ and $y$ field components are slightly different $\left(\Theta_{x} \neq \Theta_{y},\left|\Theta_{x}-\Theta_{y}\right| \ll \Theta_{x}+\Theta_{y}\right)$, the growth rates $\lambda_{1,2}$ of critical modes become different (see Fig. 5) and the amplitude Eqs. (22) become

$$
\partial_{t} A_{1}=\lambda_{1} A_{1}-4 K_{0}^{2} A_{1}\left(\left|A_{1}\right|^{2}+\left|A_{2}\right|^{2}\right)-4 \eta_{1} K_{0}^{2} A_{1}\left|A_{1}\right|^{2},
$$




$$
\partial_{t} A_{2}=\lambda_{2} A_{2}-4 K_{0}^{2} A_{2}\left(\left|A_{1}\right|^{2}+\left|A_{2}\right|^{2}\right)-4 \eta_{2} K_{0}^{2} A_{2}\left|A_{2}\right|^{2},
$$

where $\lambda_{1}>\lambda_{2}$. In this case, patterns with wave vector $\vec{q}_{1}$ only develop at, and slightly beyond, threshold. The corresponding steady state is

$$
\left|A_{1}\right|^{2}=\frac{\lambda_{1}}{4 K_{0}^{2}\left(1+\eta_{1}\right)}, \quad\left|A_{2}\right|=0 .
$$

Such patterns are stable provided that $\lambda_{2}<\lambda_{1} /\left(1+\eta_{1}\right)$. When $\lambda_{1}$ reaches $\lambda_{2}\left(1+\eta_{1}\right)$ they become unstable, and in the domain where $\lambda_{1}$ is larger than $\lambda_{2}\left(1+\eta_{1}\right)$, patterns with both wave vectors $\vec{q}_{1}$ and $\vec{q}_{2}$ are stable. Their amplitude is given by

$$
\begin{aligned}
\left|A_{1}\right|^{2} & =\frac{\lambda_{1}\left(1+\eta_{2}\right)-\lambda_{2}}{4 K_{0}^{2}\left(\eta_{1}+\eta_{2}+\eta_{1} \eta_{2}\right)}, \\
\left|A_{2}\right|^{2} & =\frac{\lambda_{2}\left(1+\eta_{1}\right)-\lambda_{1}}{4 K_{0}^{2}\left(\eta_{1}+\eta_{2}+\eta_{1} \eta_{2}\right)} .
\end{aligned}
$$

The two types of behavior (close and far from threshold) have been described in our numerical results in the preceding section. Furthermore, on increasing pumping beyond threshold, one observes a crossover between monomode patterns, with wave vectors corresponding to the maximum growth rate, and bimodal patterns. For growth rates given by Eq. (5), transition between monomodal and bimodal patterns occurs for small anisotropies $\left[\left|\left(\Delta_{x}-\Delta_{y}\right) /\left(\Delta_{x}+\Delta_{y}\right)\right| \ll 1, \mid\left(\alpha_{x}\right.\right.$ $\left.\left.-\alpha_{y}\right) /\left(\alpha_{x}+\alpha_{y}\right) \mid \ll 1\right]$ at

$$
|F|=1+\left(\frac{2+\eta_{2}}{2 \eta_{2}}\right) \frac{\left|\alpha_{x} \Delta_{x}-\alpha_{y} \Delta_{y}\right|}{\sqrt{\alpha_{x} \alpha_{y}}} .
$$

\section{CONCLUSION}

In conclusion, we have shown that standing wave intensity patterns can be generated in type-II optical parametric oscillators. They appear spontaneously in the transverse plane when there is a direct polarization coupling between the signal and idler fields, produced for example, by an intracavity quarter wave plate. Such a coupling also gives rise to two competing wavelengths in the system.

In the transient dynamical regime after the pump is switched on above its threshold value; there is a competition between two rings of unstable modes. This gives rise to transverse patterns with different wavelengths for the real and imaginary parts of the FH's fields. We have described two dynamical regimes. In the first one, which corresponds to symmetric FH's parameters, the far field is composed of two concentric rings. The real and imaginary parts of each of the FH's fields show patterns with different wavelengths. For asymmetric FH's coefficients, the dynamical regime depends on the distance from the threshold. Near threshold, the dynamical process of pattern competition leads to the dominance of a unique wavelength, selecting a transverse stripe intensity pattern with the same wavelength for the signal and idler. Far from threshold, the dynamics is equivalent to the symmetric case so that the strength of the external pump can be used to stabilize the two competing wavelengths. Amplitude equations for the vectorial (critical) modes have been derived and they confirm our numerical observations. In particular, it is worth noting that the structure of these equations reflects the vectorial nature of the fields, which introduces nontrivial couplings between the modes.

\section{ACKNOWLEDGMENTS}

We acknowledge financial support from the European Commission projects QSTRUCT (FMRX-CT96-0077) and QUANTIM (IST-2000-26019), and from the Spanish MCyT Project No. BFM2000-1108. We thank Marco Santagiustina for very helpful discussions on this topic.

\section{APPENDIX: UNIFORM PHASE-LOCKED SOLUTIONS}

In addition to the trivial uniform solution given by Eq. (3), Eqs. (1) have other uniform stationary solutions for $\Delta_{x} / \Delta_{y}>0$ considered in Ref. [24]. These are the dominant solutions when transverse effects are not taken into account. For the sake of completeness, a brief description of these solutions for $\Delta_{x, y}<0$ is given here. Equations (1) admit for $A_{x, y}=a_{x, y} \exp \left(i \phi_{x, y}\right)$ two uniform stationary solutions, which take the form

$$
\begin{gathered}
\cos \left(\phi_{d}+\xi\right)=\frac{1-\Gamma^{2}}{2|c| \Gamma}, \\
\cos \left(\phi_{s}\right)=\frac{\left(\Delta_{x}+\Gamma \Delta_{y}\right)-2|c| \Gamma \sin \left(\phi_{d}+\xi\right)}{2 K_{0} E_{0} c_{p}}, \\
a_{x}^{2}=\frac{2 c_{p} E_{0} \Gamma \sin \left(\phi_{s}\right)-1-\Gamma^{2}}{4 c_{p} \Gamma^{2}} \\
a_{y}=\Gamma a_{x},
\end{gathered}
$$

where $\Gamma^{2}=\Delta_{x} / \Delta_{y}, c_{p}=1 /\left(1-\left|c^{\prime}\right|^{2}\right)$, and $\phi_{d, s}=\phi_{x} \mp \phi_{y}$. The existence of these solutions requires for $\Gamma \neq 1$ that $|c|$ $>\left|1-\Gamma^{2}\right| /(2 \Gamma)$. This relation defines a circle in the complex plane of $c$, inside which no stationary uniform solutions exist.

The first of Eqs. (A1) indicates that these homogeneous solutions are self-phase-locked. Due to the fact that the function $\arccos$ is a multivalued function in the range $[-\pi, \pi]$, two branches of uniform phase-locked solutions exist. Each branch has a different threshold. Uniform solutions are never spontaneously observed for $\Delta_{x, y}<0$ because they have a larger threshold of instability than the solution with a finite wave number. The threshold of the uniform branch with a lower threshold is indicated by a diamond in Fig. 2. Note that this is larger than the threshold for pattern formation $\left|F_{c}\right|$ $=1$. The second branch of uniform solutions, with a larger threshold value, is unstable, and it is not observed, not even for positive FH's detunings where uniform solutions dominate and domain walls between them have been reported $[27,28]$. The homogeneous solutions described here are different from those in Fig. 11, obtained in the limit of $q_{1}=0$. 
[1] M.C. Cross and P.C. Hohenberg, Rev. Mod. Phys. 65, 851 (1993).

[2] D. Walgraef, Spatio-Temporal Pattern Formation (SpringerVerlag, New York, 1996).

[3] G. J. Valcárcel, E. Roldan, and R. Vilaseca, in Proceedings of the Euroconference: Patterns in Nonlinear Optical Systems, Alicante, Spain, 1998, special issue of J. Opt. B: Quantum Semiclassical Opt. 1, 1 (1999).

[4] F.T. Arecchi, S. Boccaletti, and P. Ramazza, Phys. Rep. 318, 1 (1999).

[5] L. Lugiato, A. Gatti, and H. Wiedemann, in Quantum Fluctuations and Nonlinear Optical Patterns, Proceedings of the Les Houches Summer School, Session 63, edited by S. Reynaud, E. Giacobino, and J. Zinn-Justin (Elsevier Science, Amsterdam, 1995).

[6] G.L. Oppo, M. Brambilla, and L.A. Lugiato, Phys. Rev. A 49, 2028 (1994).

[7] G.C. de Valcarcel, K. Staliunas, E. Roldan, and V.J. SanchezMorcillo, Phys. Rev. A 54, 1609 (1996).

[8] S. Longhi, Phys. Rev. A 53, 4488 (1996); S. Longhi and A. Geraci ibid. 54, 4581 (1996).

[9] H. Ward, M.N. Ouarzazi, M. Taki, and P. Glorieux, Eur. Phys. J. D 3, 275 (1998).

[10] P. Lodahl, M. Bache, and M. Saffman, Phys. Rev. Lett. 85, 4506 (2000).

[11] M. Santagiustina, P. Colet, M. San Miguel, and D. Walgraef, Phys. Rev. E 58, 3843 (1998).

[12] S. Trillo, M. Haelterman, and A. Sheppard, Opt. Lett. 22, 970 (1997).

[13] S. Longhi, Phys. Scr. 56, 611 (1997).

[14] M. Santagiustina, P. Colet, M. San Miguel, and D. Walgaef, Opt. Lett. 23, 1167 (1998).

[15] K. Staliunas and V. Sánchez-Morcillo, Phys. Rev. A 57, 1454 (1998)

[16] G.L. Oppo, A.J. Scroggie, and W.J. Firth, J. Opt. B: Quantum Semiclassical Opt. 1, 133 (1999); G.L. Oppo, A.J. Scroggie, and W.J. Firth, Phys. Rev. E 63, 066209 (2001).

[17] M. Le Berre, D. Leduc, E. Ressayre, and A. Tallet, J. Opt. B: Quantum Semiclassical Opt. 1, 153 (1999).
[18] M. Santagiustina, E. Hernández-García, M. San Miguel, A.J. Scroggie, and G.L. Oppo, Phys. Rev. E 65, 036610 (2002).

[19] L.A. Lugiato and F. Castelli, Phys. Rev. Lett. 68, 3284 (1992).

[20] A. Gatti et al., Phys. Rev. A 56, 877 (1997).

[21] R. Zambrini, S.M. Barnett, M. San Miguel, and P. Colet, Phys. Rev. A 65, 023813 (2002).

[22] M. Vaupel, A. Maître, and C. Fabre, Phys. Rev. Lett. 83, 5278 (1999); S. Ducci, N. Trêps, A. Maitre, and C. Fabre, Phys. Rev. A 64, 023803 (2001).

[23] E.J. Mason and N.C. Wong, Opt. Lett. 23, 1733 (1998).

[24] C. Fabre, E. Mason, and N. Wong, Opt. Commun. 170, 299 (1999).

[25] S. Longhi, Phys. Rev. A 53, 4488 (1996).

[26] G. Izús, M. Santagiustina, M. San Miguel, and P. Colet, J. Opt. Soc. Am. B 16, 1592 (1999).

[27] G. Izús, M. San Miguel, and M. Santagiustina, Opt. Lett. 25, 1454 (2000).

[28] G. Izús, M. San Miguel, and M. Santagiustina, Phys. Rev. E 64, 056231 (2001).

[29] J. Falk, IEEE J. Quantum Electron. 7, 230 (1971).

[30] R. Eckardt, C.D. Nabors, W.J. Kozlowsky, and R.L. Byer, J. Opt. Soc. Am. B 8, 646 (1991).

[31] T. Debuisschert, A. Sizmann, E. Giacobino, and C. Fabre, J. Opt. Soc. Am. B 9, 1668 (1993).

[32] H. Kimble, in Quantum Fluctuations in Quantum OpticsSqueezing and Related Phenomena, Proceedings of the Les Houches Summer School, Session 53, edited by J. Dalibard, J. Raimond, and J. Zinn-Justin (Elsevier Science, Amsterdam, 1992).

[33] H. Ward, M. Ouarzazi, M. Taki, and P. Glorieux, Phys. Rev. E 63, 016604 (2001).

[34] Equations (1) have been integrated using the algorithm described in Ref. [35]. We use a grid of $256 \times 256$ samples with grid space $\Delta x=\Delta y=0.3125$ and time step $\Delta t=0.01$. For all cases we use a flat-top super-Gaussian pump beam $E_{0}(x, y)$.

[35] R. Montagne, E. Hernández-García, A. Amengal, and M. San Miguel, Phys. Rev. E 56, 151 (1997).

[36] A. Amengual, D. Walgraef, M. San Miguel, and E. HernándezGarcía, Phys. Rev. Lett. 76, 1956 (1996). 